\title{
BRAIDED MODULES AND REFLECTION EQUATIONS
}

\author{
DIMITRI GUREVICH \\ ISTV, Université de Valenciennes \\ 59304 Valenciennes, France \\ E-mail: Dimitri.Gourevitch@univ-valenciennes.fr
}

\begin{abstract}
We introduce a representation theory of $q$-Lie algebras defined earlier in [DG1], [DG2], formulated in terms of braided modules. We also discuss other ways to define Lie algebralike objects related to quantum groups, in particular, those based on the so-called reflection equations. We also investigate the truncated tensor product of braided modules.
\end{abstract}

1. Introduction. Since the creation of the quantum groups theory enormous efforts have been made to find proper generalizations of Lie algebra structures (in spirit of super-theory) connected to the deformed enveloping algebras $U_{q}(g)$. More precisely, the following problem was considered: find an operator $[]:, V^{\otimes 2} \rightarrow V$ satisfying a twisted (quantum) version of Jacobi identity, which is somehow related with the algebras $U_{q}(g)$. Recently some hopes to find a consistent construction of such Lie algebra-like objects were connected with the so-called reflection equations (RE).

In particular, these equations participate in the construction of the bicovariant differential calculus initiated by S. Woronowicz (cf. f.e. [IP1], [IP2] and the references therein). The braided groups of S. Majid can also be treated in terms of the RE. More precisely, the braided groups can be represented as certain quotient algebras of graded quadratic algebras defined by the RE (RE algebras for short). There exists a natural but a somewhat trivial way to assign certain quadratic-linear algebras to these graded quadratic ones, which can be thought of as enveloping algebras of some Lie algebra-like objects (we represent here a version of Jacobi identity which is valid for objects of such type). However, though the RE algebras represent interesting (and, hopefully, flat in the $\operatorname{sl}(n)$-case) deformations of the function algebra on the group $S L(n)$, their further transformation into the mentioned quadratic-linear algebras is not meaningful from the deformation theory point of view (cf. Section 5).

Another way to construct q-deformed Lie algebra-like objects called braided (or q-) Lie algebras was suggested in [DG2] (the $s l(2)$-case was previously considered in [DG1]). We

1991 Mathematics Subject Classification: 17B37, 81R50.

The paper is in final form and no version of it will be published elsewhere. 
present here a construction of braided Lie algebras and make an attempt to develop their representation theory. The linear spaces, where our braided Lie algebras are represented, are called braided modules. Let us note that the family of braided modules does not form any tensor category and is a subset in the category of $U_{q}(g)$-modules (apart from the $s l(2)$-case, when any $U_{q}(s l(2))$-module can be equipped with a braided structure).

We want to emphasize that our approach to the definition of q-analogues of ordinary Lie algebras is in fact the most natural way to do it (at least for objects "living" in the category of $U_{q}(g)$-modules) although any form of Jacobi identity looking like the classical one does not exist for them (apart from the $s l(2)$-case). The reason for this is the following: the $g$-module $\operatorname{End}(g)$ contains "too many" irreducible $g$-components (cf. Remark 1 where a truncated version of Jacobi identity is discussed).

The paper is organized as follows. In Section 2 we present a construction of braided Lie algebras and compare it with some other approaches to the Lie algebra-like objects. In Section 3 we discuss a representation theory for braided Lie algebras in terms of braided modules. In Section 4 we consider some truncated version of the tensor product for them. We complete the paper with a discussion on the RE algebras.

Throughout the paper the ground field $k$ is assumed to be $R$ or $C$ and the parameter $q$ is generic. The category of $U_{q}(g)$-modules is denoted by $U_{q}(g)$-Mod.

2. Braided Lie algebras. Let us recall the construction of braided (or q-) Lie brackets introduced in [DG1] and [DG2].

Let $g$ be a simple Lie algebra. Let us assume that it is equipped with the left adjoint representation $\rho(a) z=[a, z], a, z \in g$ as an object of the category of $g$-modules. This object will be denoted by $V$. Let us consider the extension $\rho: U(g) \rightarrow \operatorname{End}(V)$ of this representation to the enveloping algebra and introduce a representation $\rho_{q}: U_{q}(g) \rightarrow$ $\operatorname{End}(V)$ deforming the previous one.

For the classical (non-exceptional) Lie algebras this deformation can be constructed as follows. Fix a vector fundamental representation $g \rightarrow \operatorname{End}(U)$ of $g$. Then the space $\operatorname{End}(U)$ can be also equipped with a $g$-module structure. Then one of the components of the decomposition of $\operatorname{End}(U)$ into a direct sum of irreducible $g$-modules $\operatorname{End}(V)=\oplus V_{\beta}$ is isomorphic to $g$ itself. Hereafter we denote the $g$-module of highest weight $\beta$ by $V_{\beta}$.

Now let us equip the space $U$ with a $U_{q}(g)$-module structure (it is well known that the action of the generators $H_{\alpha}, X_{ \pm \alpha} \in U_{q}(g)$ on the fundamental module coincides with the classical one). Let $\rho_{q}: U_{q}(g) \rightarrow \operatorname{End}(U)$ denote this representation. Then, using the coproduct operator and the antipode of the algebra $U_{q}(g)$, we equip the space $E n d(U)$ with a $U_{q}(g)$-module structure as follows:

Introduce a representation $\rho_{q}^{\text {End }}: U_{q}(g) \rightarrow \operatorname{End}(\operatorname{End}(U))$ putting

$$
\rho_{q}^{E n d}(a) M=\rho\left(a_{1}\right) \circ M \circ \rho\left(\gamma\left(a_{2}\right)\right), a \in U_{q}(g), M \in \operatorname{End}(U) .
$$

We denote the matrix product by $\circ, \gamma$ is the antipode in $U_{q}(g)$ and $a_{1} \otimes a_{2}$ is the Sweedler's notation for $\Delta(a)$.

Moreover, we deal with a coordinate representation of module elements. We consider the endomorphisms as matrices and their action is the left multiplication by these matrices. 
Let us remark that this way to equip $\operatorname{End}(U)$ with a structure of $U_{q}(g)$-module is compatible with the matrix product in it in the following sense:

$$
\rho_{q}^{E n d}(a)\left(M_{1} \circ M_{2}\right)=\rho_{q}^{E n d}\left(a_{1}\right) M_{1} \circ \rho_{q}^{E n d}\left(a_{2}\right) M_{2} .
$$

This means that the product $\circ: \operatorname{End}(U)^{\otimes 2} \rightarrow \operatorname{End}(U)$ is a $U_{q}(g)$-morphism.

Thus, we have turned the space $\operatorname{End}(U)$ into a $U_{q}(g)$-module. (Similarly we can equip the space $\operatorname{End}(U)$ with a $U_{q}(g)$-module structure for any $U_{q}(g)$-module $U$.) Decompose the space $\operatorname{End}(U)=\oplus V_{\beta}^{q}$ into a direct sum of $U_{q}(g)$-modules. One of this component can be identified with $V$ and it is equipped with a $U_{q}(g)$-module structure.

For exceptional Lie algebras this method to deform the adjoint representation does not work, but anyway for any simple Lie algebra $g$ the adjoint module $V$ can be equipped with the structure of a $U_{q}(g)$-module. Using the coproduct operator in $U_{q}(g)$, extend the $U_{q}(g)$-module structure to the space $V^{\otimes 2}$. Now decompose this $U_{q}(g)$-module into a direct sum of irreducible modules $V_{\beta}^{q}$. Then the space $V$ itself enters this decomposition with multiplicity 2 (if $g=\operatorname{sl}(n), n>2$ ) or 1 (otherwise). However, in the first case one of the modules, isomorphic to $V$, belongs to $I_{+}^{q}$ and the other belongs to $I_{-}^{q}$, where $I_{+}^{q}\left(I_{-}^{q}\right)$ denotes the q-analogue of symmetric (skew-symmetric) subspace in $V^{\otimes 2}$ (for definitions of these subspaces see, e.g., [DG2]). Let us denote the mentioned $U_{q}(g)$-modules by $V_{+}^{q}$ and $V_{-}^{q}$, respectively (the notation $V_{-}^{q}$ will be kept also in the case when the module is unique).

Then it is natural to introduce the braided Lie bracket as a $U_{q}(g)$-morphism $[,]_{q}$ : $V^{\otimes 2} \rightarrow V$ killing all $V_{\beta}^{q}$ excluding that $V_{-}^{q}$ (this property defines the bracket up to a non-trivial factor).

It is evident that this bracket is $\widetilde{S}$-skew-symmetric (i.e. [, $]_{q}=-[,]_{q} \widetilde{S}$ ) where $\widetilde{S}$ is the operator defined as follows: $\widetilde{S}=i d$ on $I_{+}^{q}$ and $\widetilde{S}=-i d$ on $I_{-}^{q}$.

The space $V$ equipped with the bracket $[,]_{q}$ will be denoted by $\bar{g}$.

Now let us introduce the enveloping algebra $U(\bar{g})$ of the braided Lie algebra $\bar{g}$. Put

$$
U(\bar{g})=T(\bar{g}) /\left\{V_{\beta}^{q}-\tau[,]_{q} V_{\beta}^{q}, \forall V_{\beta}^{q} \subset I_{-}^{q}\right\} .
$$

Hereafter we let $T(V)$ denote the free tensor algebra of the space $V$ and $\{J\}$ denote the ideal generated by the set $J \subset T(V)$. If to compare this definition to a similar definition in [DG1] and [DG2], one can see that we have introduced a factor $\tau$ in this definition. It will be discussed in Section 4 (cf. Remark 1).

Let us point out two properties of the algebra $U(\bar{g})$. First, it is evident that the product in it is $U_{q}(g)$-invariant, i.e., $a(x \circ y)=a_{1}(x) \circ a_{2}(y)$ for any $a \in U_{q}(g), x, y \in U(\bar{g})$. Second, these algebras are not the result of any flat deformation (the reader is referred to [DG2] for definitions) of the ordinary enveloping algebra since even the deformation of the symmetric algebra of the space $V$ to its q-analogue $T(V) /\left\{I_{-}^{q}\right\}$ is not flat (apart from the $s l(2)$-case), but now it does not matter for us. (However, certain quotient algebras of the algebra $U(\bar{g})$, corresponding to the $R$-matrix type orbits in terminology of [GP], can be in principle flatly deformed to their q-counterparts. The problem is to find a proper description of such "quantum orbits" in spirit of the paper [DG2]. This problem will be considered in details in other publications.) 
Let us remark that there exists a number of papers (cf. [M], [DH], [LS] and others) where Lie algebra-like objects arise as some subspaces of the quantum group $U_{q}(g)$. More precisely, the approach suggested in these papers involves searching for a finite dimensional space $L \subset U_{q}(g)$ invariant with respect to the q-adjoint operator in $U_{q}(g)$ (sometimes certain complementary conditions on $L$ are imposed, see, e.g., $[\mathrm{DH}]$ ). Then this space equipped with the bracket defined by the q-adjoint operator is regarded as a "quantum Lie algebra".

Apparently, the objects arising from this approach are close to ours. However, in our approach the space $V$ is not embedded in the quantum group. We use $U_{q}(g)$ only for a suitable description of the objects and morphisms of the category $U_{q}(g)$-Mod $\left(^{1}\right)$.

Let us also remark that in contrast with the approach developped, say, in $[\mathrm{M}]$, where certain Lie algebra-like objects (called quantum and braided Lie algebras) are introduced by means of a version of Jacobi identity our braided Lie algebras are introduced directly, by construction. They satisfy in general a very truncated form (compared with the classical one) of Jacobi identity (cf. Remark 1).

3. Braided modules. In the present Section we discuss a representation theory of the braided algebra $\bar{g}$ (essentially for $g=s l(n)$ ). Compared with quantum groups, the braided algebras look more like super- or generalized (S-)Lie algebras defined in [G] for an involutive solution $S$ to the QYBE. However, unlike the latter objects the braided Lie algebras admit only a truncated version of the representation theory. Our goal is to equip $U_{q}(g)$-modules with the structure of $U(\bar{g})$-modules but only certain of them can be "converted" into the braided ones.

DeFinition 1. We say that an $U_{q}(g)$-module $U^{q}$ is a braided module or, more precisely, a braided $\bar{g}$-module if it can be equipped with the structure of a $U(\bar{g})$-module in such a way that the corresponding map $U(\bar{g}) \rightarrow \operatorname{End}\left(U^{q}\right)$ is a $U_{q}(g)$-morphism. We also say that the classical counterpart $U=U^{1}$ of the $U_{q}(g)$-module $U^{q}$ allows braiding.

A natural way to construct braided modules is given by the following

Proposition 1. Let $U^{q}$ be a $U_{q}(g)$-module. If the decomposition $\operatorname{End}\left(U^{q}\right)=\oplus V_{\gamma}^{q}$ of the $U_{q}(g)$-module End $\left(U^{q}\right)$ into a direct sum of irreducible $U_{q}(g)$-modules is such that 1. it does not contain the modules isomorphic to $V_{\beta}^{q} \subset I_{-}^{q}$ apart from those isomorphic to $V^{q}$,

2. the multiplicity of the module $V^{q}$ is 1 ,

then $U^{q}$ can be equipped with a braided module structure (briefly, braided structure).

$\left({ }^{1}\right)$ Let us remark that our method can be applied to define a q-Lie bracket in some nonquasiclassical category, i.e., those generated by a space $V$ equipped with a non-deformational quantum R-matrix, say, that of Hecke type, possessing a non-standard Poincaré series (such quantum matrices have been constructed in $[\mathrm{G}]$ ). It would be interesting to find a q-deformation of generalized Lie brackets computed explicitly in [G] for some involutive solutions of the quantum Yang-Baxter equation. 
Pro of. By the assumption there exists a unique $U_{q}(g)$-module in $\operatorname{End}\left(U^{q}\right)$, isomorphic to $V^{q}$. Consider an $U_{q}(g)$-morphism defined up to a factor

$$
\rho: V^{q} \rightarrow \operatorname{End}\left(U^{q}\right)
$$

This map is an almost representation of the braided Lie algebra $\bar{g}$ in the sense of the following

DeFinition 2. We say that a map (1) is an almost representation of the braided Lie algebra $\bar{g}$ if it is a $U_{q}(g)$-morphism and the following properties are satisfied

1. $\circ \rho^{\otimes 2}\left(V_{\beta}^{q}\right)=0$ for all $V_{\beta}^{q} \subset I_{-}^{q}$ apart from that $V_{\beta}^{q}=V_{-}^{q}$,

2. $\circ \rho^{\otimes 2} V_{-}^{q}=\nu \rho[,]_{q} V_{-}^{q}$ with some $\nu \neq 0$

(if $\nu=\tau$, where $\tau$ will be defined below, then we have a representation).

In a more explicit form these conditions can be reformulated as follows. If the elements $\left\{b_{k, \beta}^{i, j} u_{i} u_{j}, 1 \leq k \leq \operatorname{dim} V_{\beta}^{q}\right\}$ form a basis of the space $V_{\beta}^{q} \subset I_{-}^{q}$ and analogously the elements $\left\{b_{k,-}^{i, j} u_{i} u_{j}, 1 \leq k \leq \operatorname{dim} V_{\beta}^{q}\right\}$ form a basis of the space $V_{-}^{q}$ then

$$
b_{k, \beta}^{i, j} \rho\left(u_{i}\right) \rho\left(u_{j}\right)=0 \text { if } V_{\beta}^{q} \neq V_{-}^{q} \text { and } b_{k,-}^{i, j} \rho\left(u_{i}\right) \rho\left(u_{j}\right)=\nu b_{k,-}^{i, j} \rho\left(\left[u_{i}, u_{j}\right]_{q}\right) .
$$

Let us complete the proof. The image of the composed map $\circ \rho^{\otimes 2}\left(V_{-}^{q}\right)$ is isomorphic to the $U_{q}(g)$-module $V^{q}$ since $\rho$ and $\circ$ are $U_{q}(g)$-morphisms. Such a module in the decomposition of $\operatorname{End}\left(U^{q}\right)$ is unique therefore the image of the space [, $]_{q} V_{-}^{q}=V^{q}$ with respect to the morphism $\rho$ coincides with the previous one (it suffices to show that the images of the highest weight element of the module $V_{-}^{q}$ with respect to both operators coincide up to a factor). This gives the second property of Definition 2 (the property that $\nu \neq 0$ for a generic $q$ follows from the fact that it is so for $q=1$, since in this case we have an ordinary representation $g \rightarrow \operatorname{End}(U)$ by construction).

The first property follows from the fact that $\operatorname{End}(U)$ does not contain any modules isomorphic to $V_{\beta}^{q} \subset I_{-}^{q}, V_{\beta}^{q} \neq V_{-}^{q}$. Finally, changing the scale, i.e., considering the map $\rho_{\nu}=\tau \nu^{-1} \rho$ instead of $\rho$ we get a representation of the braided Lie algebra under consideration. This completes the proof.

A natural question arises: how many $U_{q}(g)$-modules can be converted into braided ones or in other words how many $g$-modules allow braiding? An answer to this question for $s l(n)$-case is given by the following Proposition which can be proved by straightforward computations using the Young diagrams technique.

Proposition 2. Let $\omega$ be a fundamental weight of the Lie algebra $\operatorname{sl}(n)$. Then the sl(n)-modules $V_{k \omega}$ (for any $k \in N$ ) allow braiding (hereafter we denote the family of non-negative integers by $N)$. In other words, their q-analogues $V_{k \omega}^{q}$ are braided modules.

As for other simple Lie algebras $g$ it seems very plausible that a similar statement is valid for fundamental weights $\omega$ such that their orbits in $g^{*}$ are of R-matrix type (in the $\operatorname{sl}(n)$-case all fundamental weights satisfy this condition).

If this is the case, we see that "good" modules (i.e,. allowing braiding) correspond to "good" orbits (i.e. of R-matrix type) in $g^{*}$. A quantum (or braided) analogue of this correspondence may be regarded as a quantization of the ordinary orbit method (the $s l(2)$-case has been considered from this point of view in [DGR]). 
4. Truncated product of braided modules: the $s l(2)$-case. In the present Section we discuss the following problem: what is a regular way to multiply some braided modules? In the classical case, given two $g$-modules, we can equip their tensor product with a $g$-module structure by means of the ordinary coproduct operator in the enveloping algebra $U(g)$.

This procedure cannot be generalized to the braided case because of two difficulties: first, the algebra $U(\bar{g})$ does not have any Hopf structure (ordinary or braided), and second, (but in fact it is another interpretation of the first difficulty) the braided modules do not form any tensor category. However, there exists a module, namely, $V_{(k+l) \omega}$, in the tensor product $V_{k \omega} \otimes V_{l \omega}$ (where $\omega$ is a fundamental weight and $k, l \in N$ ), which can be equipped with a braided structure (we restrict ourselves to the $\operatorname{sl}(n)$-case).

Of course, the above module can be equipped with such a structure by means of the above procedure but a natural question arises: does there exist a more direct way to do this? Say, in the classical case, given a representation $\rho: g \rightarrow \operatorname{End}\left(V_{\beta}\right)$, we can equip the space $V_{k \beta} \subset V_{\beta}^{\otimes k}$ with a $g$-module structure by putting

$$
\rho_{k}(z) v=k P_{k}\left(\rho(z) \otimes i d_{k-1}\right) v, z \in g, v \in V_{k \beta},
$$

where $P_{k}: V_{\beta}^{\otimes k} \rightarrow V_{k \beta}$ denotes the natural projector and $i d_{k}$ denotes the identity operator $V_{\beta}^{\otimes k} \rightarrow V_{\beta}^{\otimes k}$. It is easy to see that the map $\rho_{k}: g \rightarrow \operatorname{End}\left(V_{k \beta}\right)$ defines a representation of the Lie algebra $g$.

It is natural to try to generalize this construction to the braided case assuming $\beta=\omega$ to be a fundamental weight (in this case both $g$-modules $V_{\beta}$ and $V_{k \beta}$ allow braiding) $\left(^{2}\right.$ ).

We call the resulting representation the truncated (since it is constructed by means of a projector) tensor product of braided modules. More generally, we consider here truncated tensor powers of the module $V_{\beta}$.

Proposition 3. Let $\rho: \overline{s l(n)} \rightarrow \operatorname{End}\left(V_{\omega}^{q}\right)$ be an almost representation of the braided Lie algebra $\overline{s l(n)}$ and let the projector $P_{k}:\left(V_{\omega}^{q}\right)^{\otimes k} \rightarrow V_{k \omega}^{q}$ be a morphism of the category $U_{q}(s l(n))-M o d$. Then the map $\rho_{k}$ defined by the formula (3) is also an almost representation of $\overline{s l(n)}$.

This proposition can be proved in the same way as Proposition 1.

Nevertheless, if the initial map $\rho$ is a representation of the braided Lie algebra $\overline{s l(n)}$ we can turn the map $\rho_{k}$ into a representation by a proper change of the factor $k$. We compute this deformed factor in the simplest case $n=2$. However, first we will consider the above factor $\tau$ in the definition of the enveloping algebra of a braided Lie algebra more precisely.

Let us consider the quantum group $U_{q}(s l(2))$, i.e., the algebra generated by the elements $H, X, Y$ satisfying the following relations

$$
[H, X]=2 X,[H, Y]=-2 Y,[X, Y]=\frac{q^{H}-q^{-H}}{q-q^{-1}} .
$$

Let us assume that the coproduct in this algebra is of the form

$$
\Delta(X)=X \otimes 1+q^{-H} \otimes X, \Delta(Y)=1 \otimes Y+Y \otimes q^{H}, \Delta(H)=H \otimes 1+1 \otimes H .
$$

$\left(^{2}\right)$ In [DG1], [DG2] a similar approach was suggested to define braided vector fields. 
Let us consider a three-dimensional space $V$ with a base $\{u, v, w\}$ equipped with the following action of the quantum group

$$
\begin{gathered}
H u=2 u, H v=0, H w=-2 w, X u=0, X v=-\left(q+q^{-1}\right) u, X w=v, \\
Y u=-v, Y v=\left(q+q^{-1}\right) w, Y w=0 .
\end{gathered}
$$

Then decomposing the space $V^{\otimes 2}$ into a direct sum of irreducible $U_{q}(s l(2))$-modules and introducing the q-Lie bracket as above we can obtain the following multiplication table for it

$$
\begin{gathered}
{[u, u]_{q}=0,[u, v]_{q}=-q^{2} M u,[u, w]_{q}=\left(q+q^{-1}\right)^{-1} M v} \\
{[v, u]_{q}=M u,[v, v]_{q}=\left(1-q^{2}\right) M v,[v, w]_{q}=-q^{2} M w} \\
{[w, u]_{q}=-\left(q+q^{-1}\right)^{-1} M v,[w, v]_{q}=M w,[w, w]_{q}=0}
\end{gathered}
$$

with an arbitrary $M$.

Then the enveloping algebra of $\overline{s l(2)}$ is defined by the following relations

$$
q^{2} u v-v u=-h u,\left(q^{3}+q\right)(u w-w u)+\left(1-q^{2}\right) v^{2}=h v,-q^{2} v w+w v=h w,
$$

where $h=\tau\left(q^{4}+1\right) M$ (this relation can be deduced from the following equality: $q^{2} u v-$ $\left.v u=\tau\left(q^{2}[u, v]_{q}-[v, u]_{q}\right)\right)$.

We fix $\tau$ in such a way that the (left) q-adjoint operators $\rho_{q}(u) z=[u, z]_{q}$ define a representation, i.e., the following relations hold:

$$
\begin{gathered}
q^{2}\left[u,[v, z]_{q}\right]_{q}-\left[v,[u, z]_{q}\right]_{q}=-\tau[u, z]_{q},\left(q^{3}+q\right)\left(\left[u,[w, z]_{q}\right]_{q}-\left[w,[u, z]_{q}\right]_{q}\right)+ \\
\left(1-q^{2}\right)\left[v,[v, z]_{q}\right]_{q}=\tau[v, z]_{q},-q^{2}\left[v,[w, z]_{q}\right]_{q}+\left[w,[v, z]_{q}\right]_{q}=\tau[w, z]_{q}, \forall z \in \bar{g} .
\end{gathered}
$$

This implies the relation $h=M\left(q^{4}-q^{2}+1\right)$ and therefore we have $\tau=1-\left(q^{2}+q^{-2}\right)^{-1}$.

We treat the latter formulae as a braided version of Jacobi identity. This means that the q-adjoint operators corresponding to the generators $u, v, w$ satisfy the same relations as the generators themselves in the enveloping algebra $U(\overline{s l(2)})$.

Rem ar $\mathrm{k} 1$. As for other simple algebras (at least $g \neq \operatorname{sl}(n), n>2$ ) we can normalize the parameter $\tau$ requiring that it satisfies the relations

$$
b_{k-}^{i, j}\left[u_{i},\left[u_{j}, z\right]_{q}\right]_{q}=\tau b_{k-}^{i, j}\left[\left[u_{i}, u_{j}\right]_{q}, z\right]_{q},
$$

where $\left\{b_{k-}^{i, j} u_{i} u_{j}, 1 \leq k \leq \operatorname{dim} V_{-}^{q}\right\}$ is a basis of the space $V_{-}^{q}$.

Unfortunately, in the general case we are unable to ensure the relations

$$
b_{k, \beta}^{i, j}\left[u_{i},\left[u_{j}, z\right]_{q}\right]_{q}=0, V_{\beta}^{q} \neq V_{-}^{q}
$$

(cf. (2)). This is the reason why the "q-adjoint representation" of a braided Lie algebra $\bar{g} \neq \overline{s l(2)}$ is not a representation at all. Summing up, we can say that the reason of this "pathology" is the following: the $g$-module $E n d(g)$ contains "too many" irreducible $g$-components (apart from the $s l(2)$-case).

Nevertheless, we treat the formula (5) as a truncated version of Jacobi identity. The term "truncated" means here that only the space $V_{-}^{q}$ (instead of the whole $I_{-}^{q}$ ) participats in these relations. 
Let us now discuss a way to modify the factor $k$ in the formula (3). Fix a base $\{a, b\}$ in a $U_{q}(s l(2))$-module $U_{1 / 2}^{q}$ such that

$$
X a=0, X b=a, H a=a, H b=-b, Y a=b, Y b=0 .
$$

Consider the map $V \otimes U_{1 / 2}^{q} \rightarrow U_{1 / 2}^{q}$ defined as follows:

$$
u \otimes a \rightarrow 0, u \otimes b \rightarrow a, v \otimes a \rightarrow q^{-1} a, v \otimes b \rightarrow-q b, w \otimes a \rightarrow q^{-1} b, w \otimes b \rightarrow 0 .
$$

It is easy to see that this map is a $U_{q}(s l(2))$-morphism. It enables us to construct an almost representation of the braided Lie algebra $\overline{s l(2)}$ according to the above procedure. This almost representation is given by

$$
\rho(u)=\left(\begin{array}{ll}
0 & 1 \\
0 & 0
\end{array}\right), \rho(v)=\left(\begin{array}{cc}
q^{-1} & 0 \\
0 & -q
\end{array}\right), \rho(w)=\left(\begin{array}{cc}
0 & 0 \\
q^{-1} & 0
\end{array}\right) .
$$

More precisely, the operators $\rho(u), \rho(v), \rho(w)$ satisfy the relations (4) with $h=h_{1}=$ $q^{3}+q^{-1}$. Consider now the almost representation $\overline{\rho_{k}}=P_{k}\left(\rho \otimes i d_{k-1}\right)$, where $P_{k}$ is the above projector in the category $U_{q}(g)$-Mod. The operators $\overline{\rho_{k}}(u), \overline{\rho_{k}}(v), \overline{\rho_{k}}(w)$ satisfy the relations (4) with a factor $h=h_{k}$. Let us compute it. Then the proper q-analogue of the factor $k$ in the formula (3) is $h_{1} / h_{k}$.

Fix the base generated by the elements $f_{1}=a^{k}, f_{2}==Y a^{k}, \ldots, f_{k+1}=Y^{k} a^{k}$ in the space $U_{k / 2}^{q} \subset\left(U_{1 / 2}^{q}\right)^{\otimes 2}$. It is easy to see that the operator $\overline{\rho_{k}}(v)$ is diagonal in this base: $\overline{\rho_{k}}(v)=\operatorname{diag}\left(v_{1}, v_{2}, \ldots, v_{k+1}\right)$. As for the operator $\overline{\rho_{k}}(u)$, it is over-diagonal.

Using the relation $q^{2} \overline{\rho_{k}}(u) \overline{\rho_{k}}(v)-\overline{\rho_{k}}(v) \overline{\rho_{k}}(u)=-h_{k} \overline{\rho_{k}}(u)$ we get $h_{k}=v_{1}-q^{2} v_{2}$. Let us compute $v_{1}$ and $v_{2}: \overline{\rho_{k}}(v) f_{1}=q^{-1} f_{1}$, i.e., $v_{1}=q^{-1}$ and

$$
\begin{gathered}
\overline{\rho_{k}}(v) f_{2}=q^{-1} P_{k}\left(a^{k-1} b+\ldots+q^{k-2} a b a^{k-2}\right)-q P_{k}\left(q^{k-1} b a^{k-1}\right)= \\
q^{-1} P_{k} f_{2}-\left(q^{k}+q^{k-2}\right) P_{k} b a^{k-1}=q^{-1} f_{2}-\left(q^{k}+q^{k-2}\right) q^{k-1} \kappa f_{2}
\end{gathered}
$$

where $\kappa=\left(1+q^{2}+\ldots+q^{2(k-1)}\right)^{-1}$. We use here the relation $P_{k} b a^{k-1}=q^{k-1} \kappa f_{2}$ which can be deduced from the following equalities

$$
P_{k} f_{2}=f_{2}, P_{k}\left(a^{i}(q a b-b a) a^{k-1-i}\right)=0, i=0, \ldots, k-2 .
$$

This gives $v_{2}=q^{-1}-\left(q^{2 k-1}+q^{2 k-3}\right) \kappa$. Finally we have $h_{k}=q^{-1}-q+q^{2}\left(q^{2 k-1}+\right.$ $\left.q^{2 k-3}\right) \kappa=\left(q^{-1}+q^{2 k+1}\right) \kappa$. Hence, we have proved the following

Proposition 4. If $\rho$ is the spin $1 / 2$ representation of the braided Lie algebra $\overline{s l(2)}$ then the map

$$
\rho_{k}=\left(q^{-1}+q^{3}\right)\left(1+q^{2}+\ldots+q^{2(k-1)}\right)\left(q^{-1}+q^{2 k+1}\right)^{-1} \rho
$$

is also a representation.

Thus, the quantity $\left(q^{-1}+q^{3}\right)\left(1+q^{2}+\ldots+q^{2(k-1)}\right)\left(q^{-1}+q^{2 k+1}\right)^{-1}$ is a proper "qanalogue" of the factor $k$ in the truncated version (3) of the tensor power.

5. Reflection equations and related objects. Let $g$ be a classical simple Lie algebra and $\mathcal{R}$ be the quantum universal R-matrix corresponding to $g$. The reflection equations $(\mathrm{RE})$ are the relations

$$
S L_{1} S L_{1}=L_{1} S L_{1} S
$$


where $L_{1}=L \otimes i d, L=\left(l_{i}^{j}\right), 1 \leq i, j \leq n, S=\sigma \rho^{\otimes 2}(\mathcal{R}), \rho: g \rightarrow \operatorname{End}(V),(\operatorname{dim} V=n)$ is the vector fundamental representation and $\sigma$ is the flip. (Let us remark that there exist other forms of the RE, see, e.g., [KSS].)

Such equations have been introduced by I. Cherednik for quantum R-matrices depending on a spectral parameter. They also occur in the construction of braided groups by S. Majid. (It is worth noting that the Majid's braided groups possess a braided Hopf structure which may be introduced by the usual matrix coproduct and a proper antipode, but we do not need this structure.)

Let $A_{0, q}$ denote the graded quadratic algebra generated by the RE (the RE algebra). More precisely, we put $A_{0, q}=T(W) /\left\{I_{-}^{q}\right\}$ where the space $W=\operatorname{Span}\left(l_{i}^{j}\right)$ is generated by the matrix elements $l_{i}^{j}$ and $I_{-}^{q}$ is the subspace in $W^{\otimes 2}$ generated by the matrix elements of $S L_{1} S L_{1}-L_{1} S L_{1} S$. Thus, the space $I_{-}^{q}$ is a q-analogue of the space of skew-symmetric tensors in $W^{\otimes 2}$ and the algebra $A_{0, q}$ is a q-analogue of the symmetric algebra $\operatorname{Sym}(W)$ of $W$.

It is well-known that this algebra becomes a $F u n_{q}(G)$-comodule if equipped with the coproduct

$$
\Delta: A_{0, q} \rightarrow A_{0, q} \otimes \operatorname{Fun}_{q}(G), l_{i}^{j} \rightarrow t_{i}^{m} \gamma\left(t_{n}^{j}\right) \otimes l_{m}^{n}
$$

where $\left\{t_{i}^{j}\right\}$ is the usual basis in the space $F_{u n}(G)$ and $\gamma$ is the antipode in $F u n_{q}(G)$ (cf. [KSS], [I1] for details). More precisely, if we equip the space $W$ and therefore the algebra $T(W)$ with a $F u n_{q}(G)$-comodule structure by means of the above coproduct then the space $I_{-}^{q}$ becomes a sub-comodule of the comodule $W^{\otimes 2}$.

Remark 2. One often considers another subspace

$$
I_{+}^{q} \subset W^{\otimes 2}, I_{+}^{q}=\operatorname{Span}\left(S L_{1} S L_{1}+L_{1} S L_{1} S^{-1}\right)
$$

which is a q-deformation of the space of symmetric tensors. The corresponding graded quadratic algebra $T(W) /\left\{I_{+}^{q}\right\}$ (together with the algebra $A_{0, q}$ ) participates in the construction of a quantum differential calculus in the algebra Fun $($ Mat $(n))$ (cf. [IP1], [IP2]). Namely, the generators of the algebra $T(W) /\left\{I_{+}^{q}\right\}$ are considered as one-sided invariant differentials on the quantum group. Meanwhile, the algebra $A_{0, q}$ is treated as one of invariant vector fields over quantum linear group.

A natural question arises: whether the quadratic graded algebra $A_{0, q}$ is a flat deformation of the ordinary symmetric algebra $\operatorname{Sym}(W)=\operatorname{Fun}(\operatorname{Mat}(n))$ ? The answer is negative for simple Lie algebras of $B_{n}, C_{n}, D_{n}$ series. As for the series $A_{n}$ it seems very plausible that the answer is positive. As far as we know there are no proofs of the flatness up to now, but a necessary condition, namely, the existence of the corresponding Poisson bracket, is fulfilled.

The mentioned bracket is well defined in the space $\operatorname{Fun}(\operatorname{Mat}(n))$ and it is quadratic with respect to the basis formed by the matrix elements $l_{i}^{j} \in F u n(\operatorname{Mat}(n))$ (for details we refer the reader to the paper [I2]). Let us only remark that the linearization of this bracket gives rise to another Poisson bracket which is linear in generators $l_{i}^{j}$. Moreover, these two brackets are compatible, i.e., any their linear combination is a Poisson bracket as well. The family of these linear combinations is called a Poisson pencil. 
Assuming the deformation $\operatorname{Sym}(W) \rightarrow A_{0, q}$ to be flat (in what follows we restrict ourselves to the $s l(n)$-case) we can consider the algebra $A_{0, q}$ as a quantization of the above mentioned quadratic Poisson bracket.

It is not difficult to construct (under the above assumption of flatness) a quantum counterpart for the whole of the Poisson pencil. It can be done by a simple change of basis in the algebra $A_{0, q}$ (in [GR] this method has been used to quantize another Poisson pencil).

To construct this quantum counterpart introduce the shift operator $S h\left(l_{i}^{j}\right)=l_{i}^{j}+h \delta_{i}^{j}$. Extending this operator to the algebra $A_{0, q}$ by multiplicativity, we get a two-parameter algebra $A_{h, q}=T(W) /\{J\}$ where the subspace $J \subset W \oplus W^{\otimes 2}$ is generated by the matrix elements of $S L_{1} S L_{1}-L_{1} S L_{1} S-h\left(L_{1} S^{2}-S^{2} L_{1}\right)$.

The latter algebra can be treated as the enveloping algebra of a Lie algebra-like object. To do this we introduce the following "generalized Lie bracket"

$$
[,]: I_{-}^{q} \rightarrow W,[,]\left(S L_{1} S L_{1}-L_{1} S L_{1} S\right)=h\left(L_{1} S^{2}-S^{2} L_{1}\right)
$$

(the latter operator is considered on each matrix element separately).

The following proposition clarifies what property of the ordinary Lie bracket is inherited by the above one.

Proposition 5. The data $\left(W, I_{-}^{q},[],\right)$ satisfy the following relations

$$
\begin{gathered}
\left([,]^{12}-[,]^{23}\right)\left(I_{-}^{q} \otimes W \cap W \otimes I_{-}^{q}\right) \subset I_{-}^{q} ; \\
{[,]\left([,]^{12}-[,]^{23}\right)\left(I_{-}^{q} \otimes W \cap W \otimes I_{-}^{q}\right)=0 .}
\end{gathered}
$$

Proof. Consider an element $Q$ of the space $I_{-}^{q} \otimes W \cap W \otimes I_{-}^{q}$. By definition it can be represented as $Q=\sum f_{i} k_{i}=\sum m_{j} g_{j}$ where $f_{i}, g_{i}$ are certain elements from $I_{-}^{q}$ and $k_{i}, m_{j}$ belong to $W$. Apply the operator [, $]^{12}-[,]^{23}$ to the element $Q$. We have

$$
\left([,]^{12}-[,]^{23}\right) Q=\sum\left(\left[f_{i}\right] k_{i}-m_{i}\left[g_{i}\right]\right)=\sum\left(f_{i} c_{i}-d_{i} g_{i}\right)
$$

where the elements $c_{i}, d_{i} \in k$ are defined as follows: $c_{i}=(S h-i d) l_{i}, d_{i}=(S h-i d) m_{i}$ (to get the latter relation it suffices to apply the operator $S h$ to $0=Q-Q=\sum f_{i} k_{i}-\sum m_{j} g_{j}$ and to gather the elements containing $h)$. This proves the relation (6). To get (7) it suffices to use the relation $\sum\left[f_{i}\right] c_{i}=\sum d_{i}\left[g_{i}\right]$ (we can prove it applying the operator $S h$ to $Q-Q$ and gathering the terms with $h^{2}$ ).

Remark 3. Let us remark that the formulae (6) and (7) represent the most general form of Jacobi identity connected to the deformation theory. It was suggested by A. Polishchuk and L. Positcelski who have proved a version of the PBW theorem (under the assumption of "Koszulity" of the corresponding graded quadratic algebra) for Lie algebra-like objects of such type (cf. [BG] where a slight generalization of the mentioned result is given).

Using this result it is possible to prove the following statement. Let a family of graded quadratic algebras $A_{0, q}=T(V) /\left\{I^{q}\right\}$ be a flat deformation of the symmetric algebra $\operatorname{Sym}(V)$ of a linear space $V$ and let a bracket [, ] : $I^{q} \rightarrow V$ satisfy the above version of Jacobi identity with $I_{-}^{q}=I^{q}$. Then the corresponding enveloping algebra (with a factor $h$ at the bracket) $A_{h, q}=T(V) /\left\{I^{q}-h[,] I^{q}\right\}$ is also a flat two parameter deformation. 
Let us also remark that the above bracket [, ] is defined only on the space $I_{-}^{q}$ but we can extend it to the whole $W$ in a natural way by setting $[,] I_{+}^{q}=0$. The extended data $\left(V, I_{-}^{q}, I_{+}^{q},[],\right)$ of such type (with a complementary condition that the quadratic algebra $T(V) /\left\{I^{q}\right\}$ is Koszul) have been called in [DG2] generalized Lie algebras.

Nevertheless, in the case under consideration we do not need the above mentioned generalization of the PBW theorem since the deformation $A_{0, q} \rightarrow A_{h, q}$ is flat by construction and assuming the deformation $\operatorname{Sym}(W) \rightarrow A_{0, q}$ to be flat we automatically have a flat two parameter deformation $\operatorname{Sym}(W) \rightarrow A_{h, q}$.

Let us also remark that the Jacobi identity (6), (7) differs from that given by the formula (5). If the former one is connected to the deformation theory of quadratic algebras the latter one is rather motivated by representation theory of the braided Lie algebras.

Thus, we have defined generalized Lie algebras related to the RE (assuming the flatness conjecture to be true, it is easy to show that the graded quadratic algebra $A_{0, q}$ is Koszul for a generic $q$ ). There exists a number of papers where these Lie algebra-like objects are considered as proper q-deformations of ordinary Lie algebras, taking the following fact into consideration. The above mentioned linear Poisson bracket coincides with the $g l(n)$ Lie bracket on the generators $l_{i}^{j}$ (cf. [KSS], [I2]). This is also a reason why the elements of the algebra $A_{0, q}$ are often treated as one-sided invariant vector fields.

However, in contrast to the braided Lie algebras considered in the previous Sections these objects are constructed in a trivial (from the deformation theory point of view) way and for this reason they do not give rise to any interesting generalization of the Lie algebra structure.

Let us remark that the space $W\left(\operatorname{dim} W=n^{2}\right)$ can be decomposed into a direct sum of two irreducible Fun $_{q}(S L(n))$-comodules of dimensions 1 and $n^{2}-1$ respectively. This decomposition coincides in fact with that described in Section 2 (note that there exists a natural way to equip any $\operatorname{Fun}_{q}(S L(n))$-comodule with a $U_{q}(s l(n))$-module structure).

We can treat the latter component as a q-deformation of $\operatorname{sl}(n)$. However, the algebras $A_{h, q}$ (even if $h=1$ ) cannot be restricted to this component (to see this, it suffices to show that the above mentioned quadratic Poisson bracket cannot be restricted to $\operatorname{sl}(n))$. So, if we want to get a Lie algebra-like object which is well-defined on the latter component, we come to the above construction of a braided Lie bracket.

Acknowledgments. The author thanks G. Arutyunov, J. Donin, A. Isaev, P. Pyatov, S. Majid and S. Shnider for helpful discussions. The work was partially supported by grant ISF MBI000.

\section{References}

[BG] A. Braverman, D. Gaitsgory, Poincaré-Birkhoff-Witt theorem for quadratic algebras of Koszul type, hep-th/9411113.

[DH] G. Delius, A. Hüffmann, On quantum algebras and quantum root systems, q-alg/ 9506017.

[DG1] J. Donin, D. Gurevich, Braiding of the Lie algebra sl(2), Amer. Math. Soc. Transl. (2) 167 (1995), pp. 23-36. 
[DG2] J. Donin, D. Gurevich, Quantum orbits of R-matrix type, Lett. Math. Phys. 35 (1995), pp. 263-276.

[DGR] J. Donin, D. Gurevich, V. Rubtsov, Quantum hyperboloid and braided modules, q-alg/9511014.

[G] D. Gurevich, Algebraic aspects of the quantum Yang-Baxter equation, Leningrad Math.J. 2 (1991), pp. 801-828.

[GP] D. Gurevich, D. Panyushev, On Poisson pairs associated to modified R-matrices, Duke Math. J. 73 (1994), pp.249-255.

[GR] D. Gurevich, V. Rubtsov, Quantization of Poisson pencils and generalized Lie algebras, Teor. i Mat. Phys. 103 (1995), pp. 476-488.

[I1] A. Isaev, Interrelation between Quantum Groups and Reflection Equation (Braided) Algebras, Lett. Math. Phys. 34 (1995), pp. 333-341.

[I2] A. Isaev, Quantum Groups and Yang-Baxter Equation, Phys. Part. Nucl. 26 (1995) n 5, pp. 501-526 (Russian).

[IP1] A. Isaev, P. Pyatov, $G L_{q}(N)$-covariant quantum algebras and covariant differential calculus, Phys. Let. A 179 (1993), pp.81-90.

[IP2] A. Isaev, P. Pyatov, Covariant differential complex on quantum linear groups, J. Phys. A: Math. Gen. 28 (1995), pp. 2227-2246.

[KSS] P. Kulish, R. Sasaki, C. Schwiebert, Constant Solution of Reflection Equations and Quantum Groups, hep-th/9205039.

[LS] V. Lyubashenko, A. Sudbery, Quantum Lie algebras of type $A_{n}$, q-alg/9510004.

[M] Sh. Majid, Quantum and braided Lie algebras, JGP 13 (1994), pp.307-356. 